\title{
Catching the light curve of flaring GRBs: The opportunity offered by scanning telescopes
}

\author{
G.M. Beskin ${ }^{1}$, V. Plokhotnichenko ${ }^{1}$, C. Bartolini ${ }^{2}$, A. Guarnieri ${ }^{2}$, N. Masetti ${ }^{3}$, A. Piccioni ${ }^{2}$, A. Shearer ${ }^{4}$, \\ A. Golden ${ }^{4}$, and G. Auriemma ${ }^{5}$ \\ 1 Special Astrophysical Observatory, Russia \\ 2 Dipartimento di Astronomia, Università di Bologna, via Zamboni 33, I-40126 Bologna, Italy \\ 3 Istituto Te.S.R.E., CNR, Bologna, Italy \\ 4 University of Galway, Ireland \\ ${ }^{5}$ Gruppo di Fisica Astroparticellare, Università della Basilicata, Potenza, Italy
}

Received December 18, 1998; accepted July 1, 1999

\begin{abstract}
We discuss the project of a wide field optical telescope equipped with panoramic detectors in order to catch an optical transient (OT) simultaneously with Gamma-Ray Burst (GRB). This telescope scans the gamma-ray telescope field of view, and with $1 \mathrm{~m}$ mirror for $1 \mathrm{~s}$ of integration time would detect OTs up to $16 \mathrm{mag}$; the probability of covering the GRB location in a field of $20^{\circ}$ is $12 \%$. The detection limit could be raised and the probability of catching OTs could be over 90\% using a system of special telescopes with wide aperture and large collecting area.
\end{abstract}

Key words: telescopes, instrumentation: detectors, gamma-rays: bursts

\section{Introduction}

The detection of the faint optical afterglows in the last two years has not solved the mystery of GRBs. The optical behaviour of GRBs in the very early onset of the $\gamma$-ray emission and earlier is unknown because no simultaneous observations have been obtained up to now over 10 mag (Bernas et al. 1998). Even the recently discovered optical emission from GRB 990123 at the level of 9-12 mag was detected by ROTSE only $22.18 \mathrm{~s}$ after the onset of the burst (Akerlof et al. 1999).

The hope of finding some signature of the nature of the GRB parent bodies probably rests with the observation of OTs, possibly simultaneously with $\gamma$-ray emission. The difficult observations of light flashes, unpredictable in

Send offprint requests to: bartolini@astbo3.bo.astro.it time and position, started on the basis of the archival astroplates analysis (Greiner \& Wenzel 1990, and references therein).

Since the typical duration of a GRB is as short as a few seconds, with a very fast rising time and a temporal evolution characterized by a strong stochastic variability, an "ad hoc" projected telescope should have high time resolution, good sensitivity and obviously a very wide field of view. The request of spatial resolution is less important because a precise localization can be devolved to the observations of the afterglow. Practically no OT monitoring project combining these conditions exists.

For these reasons we propose the project of an optical telescope scanning the field of view of a $\gamma$-telescope during its observation and accumulating information about faint OTs independently from GRBs detection (and data about precise GRBs position).

\section{New instrumentation to solve old problems}

To achieve the goal of a simultaneous detection of an optical- $\gamma$-ray event the optical telescope must be already observing that field from where the GRB will be received. To do this the minimal requisite is a wide field optical telescope scanning the sky area under observation by $\gamma$-ray satellite. As an example we now analyze the following configuration: i) telescope: mirror diameter $D=1 \mathrm{~m}$, focal ratio $f=1: 1$, size of field $l=3^{\circ}$, scale factor $f s=206^{\prime \prime} \mathrm{mm}^{-1}$; ii) detector: two $2 \times 2$ CCD arrays, each CCD featuring $25 \times 25 \mathrm{~mm}$ active area, $40000 \mathrm{re}-$ binned pixels (with pixel size $d=300 \mu \mathrm{m}$, corresponding to about $70^{\prime \prime}$ in the focal plane), quantum efficiency $\eta=40 \%-80 \%$ in the range $4000-8000 \AA$, read-out time $1 \mathrm{~s}$ (equal to the exposure time, to allow double buffer image acquisition by switching between the two arrays). 
We now evaluate the detection limit and the catching probability when such a telescope is scanning the $\gamma$ detector field of view. We assume $1 \mathrm{~s}$ exposures and a $0.1-0.5 \mathrm{~s}$ shifting time from one position to the next.

\section{One-telescope equipment detection limit}

Assuming standard flux calibration (Fukugita et al. 1995), we can set the detection limit in the $R$ band. The number of collected electrons per s per pixel is

$N=5.710^{4}\left(\frac{D}{1 \mathrm{~m}}\right)^{2} \frac{\eta}{0.6}\left(\frac{d}{70^{\prime \prime}}\right)^{2} 10^{-0.4\left(b_{R}-20.5\right)}$.

In the context of our example? is normalized to 1 for a sky brightness magnitude $b_{R}=20.5 \operatorname{arcsec}^{-2}$.

Assuming Poisson statistics for the photonic signal the limit magnitude $m_{R}$ for the detection of the OT is

$m_{R}=5.78+0.5 b_{R}+2.5 \cdot \log \left[\frac{210 D}{\alpha d}\left(\frac{\eta \tau}{0.6}\right)^{\frac{1}{2}}\right]$,

where $\alpha$ is the detection level and $\tau$ the exposition time; units are: $D$ in $\mathrm{m}, d$ in $\operatorname{arcsec}, \tau$ in s. For the parameters of the example this corresponds to $m_{R} \sim 16$. Concerning the data flux, taking into account displacement times, 10 hours of continuous accumulation would produce 24000 frames, i.e. about 1.92 Gbytes/night.

\section{Probability of detection}

For OTs brighter than the limit magnitude the probability of detection depends only on the fraction of the overall field of view of size of the $\gamma$-ray satellite monitored by the optical telescope during the transient event. With the assumed parameters for the telescope and typical duration of GRBs and $20^{\circ}$ size of the field the probability $P$ is

$P=0.12 \cdot\left(\frac{L}{20^{\circ}}\right)^{-2} \cdot\left(\frac{l}{3^{\circ}}\right)^{2} \cdot\left(\frac{T}{7 \mathrm{~s}}\right) \cdot\left(\frac{t_{l}}{1 \mathrm{~s}}\right)^{-1}$,

where $T$ is the GRB duration and $t_{l}$ is the telescope scanning time of the field $l$.

\section{A new non-conventional approach}

Now we discuss the possibility of increasing the probability of detection and of gaining sensitivity. Concerning the first problem we should use telescopes with larger fields of view and, to raise the detection sensitivity, larger collecting areas. In our opinion the best way to solve the above problems is to use large mirrors but - this is a major point - the high resolution typical of optical telescopes is unnecessary. There are several kinds of such instruments: Cherenkov telescopes currently operating or under construction (WOIT: Weeks 1988; CAT: Punch 1995; CANGAROO: Lamb 1997; SUBURU: Tanimori et al.
1995; MAGIC: Lorenz 1995), radioparaboloids and military radars (covered by mirror film). Cherenkov telescopes are large mirror or multimirror devices used to take images of Cherenkov light showers from gamma-ray primaries in VHE and UHE energy bands (GraniteIII, Lamb et al. 1995); they have wide field and their resolution, not suitable for conventional astronomical image acquisition, could attain with little effort $1^{\prime}$, enough for our purpose.

Assuming for the Cherenkov telescope $D=10 \mathrm{~m}$, focal ratio $1: 1$, size of focal plane $1 \mathrm{~m} \times 1 \mathrm{~m}$, pixels size $=5 \mathrm{~mm} \times 5 \mathrm{~mm}=1^{\prime} \times 1^{\prime}$, we get a detection limit at $m_{R}=18.5$.

Widening the field of view from $3^{\circ}$ to $6^{\circ}$ square degrees (Lamb 1997) and at a slew rate of $6^{\circ}$ per second the probability of detection of an OT of typical duration would be $40 \%$ if the telescope stands during each integration and of $87 \%$ if the telescope continuously scans the field (but with obvious software complications).

The use of arrays of fast Panoramic Photoelectric Detectors, as MAMA (Timoty 1988) and EBCCD (CERN 1997) or of multianode PMT (Hamamatsu catalogue 1999) to build the imaging device to be put on the focal plain canister could allow the observation of the OT fine time structure (flashes of about $10^{-4} \mathrm{~s}$ have been detected in the light curves of GRBs).

Acknowledgements. This investigation was supported by the Russian Fund of Fundamental Researches (grant 98-0217570), by the Educational-Scientific centre "Cosmion", by the Italian Ministry of Foreign Affairs, and by the University of Bologna (Funds for selected research topics).

\section{References}

Akerlof C., et al., 1999, GCN 205

Bernas M., et al., 1998, $4^{\text {th }}$ Huntsville Symposium, 1998, p. 864 CERN, 1997, project status report CERN/LHCC97-38

Fukugita M., et al., 1995, PASP 107, 945

Greiner J., Wenzel W., 1990, Los Alamos Workshop Proc., p. 119

Hamamatsu catalogue, 1999

Lamb R.C., et al., 1995, Proceedings of the Padova Workshop on TeV Gamma-Ray Astrophysics, p. 386

Lamb R.C., 1997, Ital. Phys. Soc. Conf. Proc. 57, 256

Lorenz E., 1995, Proceedings of the Padova Workshop on TeV Gamma-Ray Astrophysics, p. 277

Punch M., 1995, Proceedings of the Padova Workshop on TeV Gamma-Ray Astrophysics, p. 356

Tanimori T., et al., 1995, Proceedings of the Padova Workshop on TeV Gamma-Ray Astrophysics, p. 316

Timoty J.G., 1988, Ninth Santa Cruz Summer Workshop in Astronomy and Astrophysics, July 13-24, 1987, Lick Observatory: Instrumentation for Ground-Based Optical Astronomy, Present and Future. Springer-Verlag, p. 522

Weeks T.C., 1988, Physics Reports 160: Very High Energy Gamma-Ray Astronomy. North-Holland, Amsterdam, p. 103 